\title{
Efficient PF-POF external LPG index Transducer
}

\author{
J. Castrellon-Uribe ${ }^{1,2}$, M. Lomer ${ }^{1 *}$, H.S.G. Roufael ${ }^{1}$, and J.M. Lopez-Higuera ${ }^{1}$ \\ ${ }^{1}$ Photonics Engineering Group, Cantabria University, Av. De los Castros s.n. 39005 Santander, Spain \\ ${ }^{2}$ Centro de Investigación en Ingeniería y Ciencias Aplicadas, CIICAp, Universidad Autónoma del \\ Estado de Morelos, UAEM, Av. Universidad 1001, Col. Chamilpa, Cuernavaca Morelos, México. \\ *lomerm@unican.es
}

\begin{abstract}
The fabrication of long-period grating in multimode polymer optical fibers for low refractive index for liquids sensing is reported in this paper. Good sensitivity to sugar concentration on water is obtained.

OCIS codes: (060.2340) Fiber optics components; (060.2370) Fiber optics sensors
\end{abstract}

\section{Introduction}

Plastic optical fibers (POF) have been shown to be key components of short distance communications and sensor applications. In recent years there have been numerous studies and applications have been numerous devices. Particular attention has been focused on fiber Bragg gratings (FBG) in single-mode and multimode fibers. Unlike silica fibers, where FBG is based on UV photosensitivity of germanium-doped silica, the FBGs in POF have been made previously by doping the core based in polymethil-methacrylate (PMMA) with a photosensitive material. That is not commercially available materials [1]. However, in recent years there have been important advances in new structures and new materials for POF, particularly fibers with cores perfluorinated (PF), which is an excellent material and have low loss in the region of the visible and infrared spectrum. Good that these fibers have been manufactured to work in high-speed communications [2-3]. They can be interesting in applications in sensor technology, particularly because of its low value of core refractive index.

This paper first presents the fabrication of LPG's in PF-GI-POF using a UV pulsed laser $355 \mathrm{~nm}$. The fibers used in this work is commercially available and has a ratio of diameter core/cladding of 50/500 and 120/500 $\mu \mathrm{m}$. The LPGs are performed on the lateral side of the cladding fiber and written point by point. This fabrication method allows the modulation index variation of the cladding controlling the grooves depth, where the experimental results show a greater sensitivity to the influence of the external environment. Such LPG's structures would have potential applications in the sensing of liquids using PF-GI-POF.

\section{Fabrication LPG in perflurinated polymer optical fiber}

The development of the perfluorinated (PF) amorphous polymer-based GI POF [3-4] initially used to improve the high speed communications with POF have a low core refractive index relative to PMMA-POF [5]; this can be very interesting for applications in sensor technology. Indeed, the transmission spectrum of such fiber in the present low ordered in the visible and near infrared extending to $1300 \mathrm{~nm}$. The refractive indices of core and cladding are about 1.35 and 134, respectively, values compatible with many liquid substances about refractive index of water. For example, sugar or salt concentrations. Furthermore, fibers are currently commercially available. In Fig. 1 the transmission spectrum of the PF-GI-POF before writing of grating is shown.

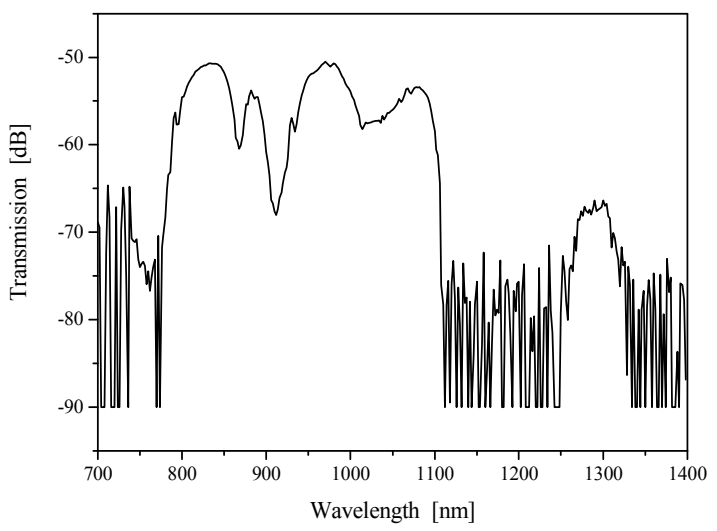

Fig. 1. Transmission spectrum of the perfluorinated polymer optical fiber. 
The experimental setup of fabrication consists of a UV pulsed laser wavelength $355 \mathrm{~nm}$ obtained from the third harmonic of Nd:YAG laser. The pulses have a frequency of $10 \mathrm{~Hz}$. The laser beam is focused by a lens on a lateral side of the cladding fiber. Writing the grating on the lateral side of the fiber is performed point by point and longitudinal displacement along the axis. LPG's are characterized online by a white light source and optical spectrum analyzer. In this work we have used two types PF-GI-POF, GI-POF50 and GI-POF1200 and manufactured by Chromis Fiberoptics ${ }^{\circledR}$ with a diameter ratio core/cladding of $50 / 500 \mu \mathrm{m}$ and $120 / 500 \mu \mathrm{m}$, respectively. Transmission spectra before and after fabrication under the same conditions for the two fibers are shown in Fig. 2. After manufacturing losses increase when the fiber core is higher, also increases the rejection band of the LPG. The resonant length is $910 \mathrm{~nm}$ and the band rejection are $12 \mathrm{dBs}$ (Fig. 2(a)) and $14 \mathrm{dBs}$ (Fig. 2(b)). This result is logical that the groove made in the cladding is closer to the fiber core $120 / 500$ with respect to the fiber 50/500.
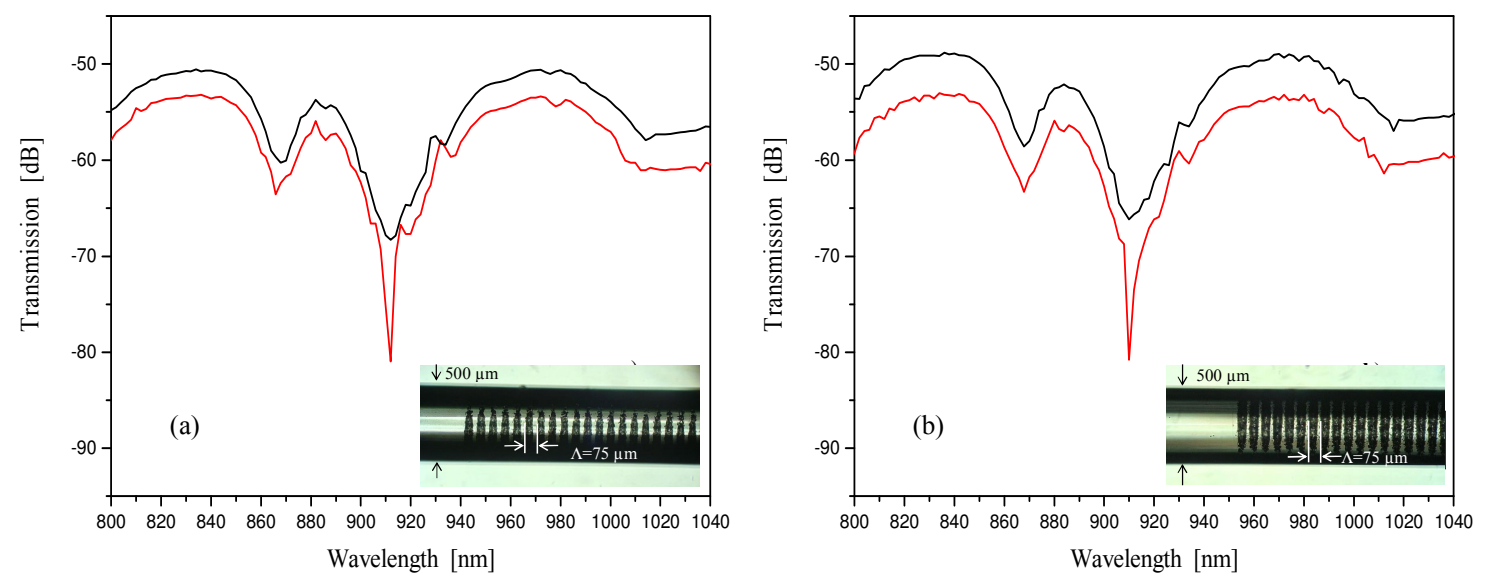

Fig. 2. Transmission spectrums of the PF-GI-POF before and after grating fabrication, optical microscope images of the structure of the LPG fabricated a) $50 / 500 \mu \mathrm{m}$ and b) $120 / 500 \mu \mathrm{m}$ core/cladding fiber. The period of the grating is $75 \mu \mathrm{m}$ and a length of $7.5 \mathrm{~mm}$ of LPG.

\section{Application to the measurement liquid concentration.}

The LPG made asymmetrically have proved very sensitive to the influence of the external environment with respect to the LPG's made in the fiber core or symmetrically in the fiber cladding [5]. The LPGs shown in Fig. 2 are used to measure this influence immersing the LPG at different concentrations of sugar and salt in water. The experimental results show the displacement of the resonance wavelength. For example, when the LPG is immersed in water $(n=1.33)$, the resonance peak is shifted to lower wavelengths as the sugar or salt concentration increases, the resonance peak is shifted to higher wavelengths. The shifted are of $3 \mathrm{~nm}$ and $6.5 \mathrm{~nm}$ when the water contains $5 \mathrm{~g} / 1$ and $10 \mathrm{~g} / \mathrm{l}$, respectively. The results agree with the predictions of reference [5].

\section{Conclusion}

The fabrication of LPG's on a PF-GI-POF is reported in this paper. The grating is written point by point on a lateral side of the cladding of the fiber without affecting the fiber core. The concentration's measure of sugar or salt in water has been used to show high sensitivity to the external environment of the LPGs. An additional advantage of the LPGs in PF-GI-POF is the low refractive index of the fiber. The low refractive index of PF-GI-POF fibers enable the optimization of the proposed structure to measure low refractive indices of the soft-matters.

Acknowledgement: this work was supported by the MCYT (Spain), project CICYT: TEC2010-20224-C02-02 and CONACyT (Mexico) by sabbatical stay at the University of Cantabria, file: 186243.

\section{References}

[1] Xiong, Z., Peng, G.D., Wu, B., Chu, P.L., "Highly Tunable Bragg Gratings in Single-Mode Polymer Optical Fibers", IEEE Photon. Technol. Lett., 11, 352-354 (1999).

[2] Liu,H.Y., Peng, G.D., Chu, L.P., “Thermal stability of gratings in PMMA and CYTOP polymer fibers”, Opt. Commun. 204, 151-156 (2002).

[3] Yoshihara, N., "Low-loss, high-bandwidth fluorinated POF for visible to 1.3-mm wavelength," in Proc. Optic. Fiber Conf. (OFC'98), San Jose, CA, Paper ThM4 (1998).

[4] Ishigure, T., Koike, Y., and Fleming,J.W., "Optimum Index Profile of the Perfluorinated Polymer-Based GI Polymer Optical Fiber and Its Dispersion Properties", J.Lightwave Technol., 18, pp. 178-184, (2000).

[5] Zhu, T., Rao, Y-J., Wang, J-L., and Song, Y., “A highly sensitive fiber-optic refractive index sensor based on an edge-written long-period fiber grating”, IEEE Photon. Technol. Lett., 19, 1946-1948 (2207). 